\title{
Editorial
}

\section{Personal Fellowships in Otolaryngology}

A Register of Training Fellowships in Otorhinolaryngology - Head and Neck Surgery in the United Kingdom should be established

The new arrangements for training which have been proposed under the Calman recommendations mean that opportunities for subspecialization need to be created and funded. It is unrealistic to expect that most trainees will have a satisfactory opportunity to gain such experience within their own hospitals and many will therefore need to travel.

Where is this training to take place and how are some trainees to be selected for special additional training over and above what might be expected within the course of a routine training programme?

At present senior trainees are generally encouraged to make their own arrangements to travel abroad and funds are secured from charitable foundations and other means. A mechanism for securing further training in this country is currently hard to identify although there are probably many consultants who are in a position to offer further special or subspecialized experience in aspects of ENT Surgery.

Consultants who believe that they are in such a position might seek to establish visiting fellowships of varying duration. The success or failure of the fellowship would soon come to be judged by the fellows themselves who would competitively oversubscribe the good ones and ignore the poor ones, thus ensuring that the system was to some extent self regulating.

Personal fellowships with named individual consultants are likely to work best. Some fellowships will be in famous teaching hospitals where there is already an entourage of trainees and some will be in lesser known hospitals. Historically much of the most innovative otorhinolaryngological surgery in this country has taken place away from the great teaching institutions and to some extent training opportunities may have been lost. The Personal Fellow, free of service commitment, might also be in a position to take advantage of training opportunities in private hospitals. A close personal relationship between the fellow and the consultant may come to be seen as a major advantage of the system as it develops.

Financial support for the Fellowships should come from the budget of the Regional Postgraduate Deans. A general agreement on reciprocity of salaries would mean that few regions would be net losers.

A Register of Fellowships should be created under the aegis of an appropriate body such as the Specialist Advisory Committee of The Royal Colleges of Surgeons who might chose to carry out periodic inspections.

The new arrangements for the salaries of trainees to be controlled by the Regional Deans means that an opportunity for a genuine innovation in British surgical training has arisen. The introduction of such a system of personal fellowships to augment the current training programmes would be welcomed by the present generation of trainees and secure a mechanism for the subspecialization that is envisaged for some of the trainees in the anticipated Unified Training Grade.

MR PETER PRINSLEY

Sec. of the Association of Otolaryngologists in Training

\section{Personal Fellowships in training}

It would be helpful for the trainee in future to have access to a Register of Training Fellowships as suggested. The Education and Training Committee of the British Association of Otorhinolaryngology-Head and Neck Surgery (BAOL) is looking to do this for overseas Fellowships, but the creation of a Personal Fellowship in the UK as part of advanced subspeciality training would be a new venture and could give a unique opportunity for advanced subspeciality training during the sixth year of Higher Surgical Training. The Postgraduate Deans in England and Wales make available only half the training budget but as no or very little service commitment would be undertaken by the trainee it may be difficult to persuade a Trust to pay for the other half of the training. When all the training budget is held by the Postgraduate Dean, as is at present in Scotland, it is hoped that Deans would be prepared to look at cross regional funding.

Consultants who are recognized for their expertise and teaching on a particular subspeciality interest will already by default be committed to the training of one or two Higher Surgical Trainees already allocated to the department, so it would be essential that a Personal Fellowship post was not regarded as an extra post taking away clinical work from other trainees already in the programme. This may produce a problem of continuity with regard to established rotations and a Personal Fellowship would have to be arranged well in advance and would be unlikely to be on a regular basis. Attendance of a trainee at a private hospital for teaching is not new; already there is a precedence for this as a private hospital has already been recognized for Higher Surgical Training by the SAC in Plastic Surgery. Even the most famous teaching hospitals do not have an entourage of trainees as suggested as the numbers for both teaching and non teaching hospitals is strictly controlled by the Department of Health manpower recommendations and with educational approval by the SAC.

A Personal Training Fellowship would need to be planned on a national basis and the number of Fellowships carefully regulated so that the long term requirements of the speciality were met and, at the same time, there was a reasonable opportunity for the trainee to succeed in a Consultant appointment to that subspeciality as a suitable post may become available only every four or five years.

There is undoubtedly a need to develop the opportunities for subspeciality training to the full advantage of the trainees, and the idea of creating a Personal Fellowship during the latter part of Higher Surgical Training draws attention to this need.

D.w. 